\title{
The dose can make the poison: lessons learned from adverse in vivo toxicities caused by RNAi overexpression
}

\author{
Dirk Grimm
}

\begin{abstract}
For the past five years, evidence has accumulated that vector-mediated robust RNA interference (RNAi) expression can trigger severe side effects in small and large animals, from cytotoxicity and accelerated tumorigenesis to organ failure and death. The recurring notions in these studies that a critical parameter is the strength of RNAi expression and that Exportin-5 and the Argonaute proteins are rate-limiting mammalian RNAi, strongly imply dose-dependent saturation of the endogenous miRNA pathway as one of the underlying mechanisms. This minireview summarizes the relevant work and data leading to this intriguing model and highlights potential avenues by which to alleviate RNAi-induced toxicities in future clinical applications.
\end{abstract}

Keywords: RNAi, shRNA, miRNA, viral vector, toxicity, saturation

\section{Background}

Since the seminal 1998 report of RNA interference (RNAi) in nematodes [1], the ascent of RNAi technologies from a curious phenomenon in worms to a widely and routinely used surrogate genetic tool in higher eukaryotes, as well as one of our most promising therapeutic modalities, has been nothing short of meteoric. Ironically, though, in the same year, 2006, that the rise of RNAi temporarily culminated in the Nobel Prize for its pioneers Andrew Fire and Craig Mello, Mark Kay's group published a startling study reporting fatal side effects from abundant RNAi expression in the livers of adult mice [2]. Since then a series of further studies in various species and tissues have solidified the original idea that one crucial mechanism underlying the observed in vivo toxicities or fatalities is adverse saturation of the endogenous miRNA machinery by ectopic RNAi triggers. Herein I briefly review these papers and findings before highlighting key lessons that we can learn and new avenues that we can now take.

\footnotetext{
Correspondence: dirk.grimm@bioquant.uni-heidelberg.de
} Cluster of Excellence CellNetworks, Department of Infectious Diseases, Virology, University of Heidelberg, BioQuant BQ0030, Room 502a, Im Neuenheimer Feld 267, D-69120 Heidelberg, Germany
From observations of dose-dependent in vivo RNAi toxicities...

The 2006 Grimm et al. study [2] came as a surprise to the field, as the wealth of previous reports had proved RNAi's superior efficacy and thus fostered a rapid translation of RNAi technologies from bench to bedside. What was so different in this particular work was the unique combination of (1) an utmost potent viral RNAi delivery vector (self-complementary adeno-associated virus serotype 8 (scAAV8)), (2) a powerful promoter (U6, one of the strongest known RNA polymerase III promoters) driving small hairpin RNA (shRNA) expression and (3) delivery of high vector doses (directly into the hepatic circulation in some animals) [2]. This experimental setup not only ensured complete liver transduction in the injected mice but also introduced, on average, thousand RNAi expression templates into each hepatocyte, likely resulting in the transcription of hundreds of thousands of shRNA molecules per cell.

Unsurprisingly, at least in retrospect, such massive overloading of the cells with exogenous RNAi inducers was most likely more than what their endogenous RNAi machinery could handle. This is evidenced by the study's finding that more than 20 different abundantly expressed shRNAs caused substantial hepatotoxicities and eventual fatalities, regardless of the presence or 
absence of targets and without other detectable adverse reactions, such as immune responses. Notably, shRNA overexpression and toxicity correlated with dysregulation of hepatocellular miRNAs, implying competition of shRNAs and miRNAs for rate-limiting factors and substantiating the idea that saturation of the liver RNAi machinery was a major cause of toxicity.

Subsequently, a series of other studies made very similar observations in mouse livers and came to a comparable conclusion. This includes a recent report by Borel and colleagues [3], who also used ScAAV8 for in vivo shRNA transduction and noted viral dose-dependent hepatotoxicities in mice, which were evidenced by increases in plasma transaminases and animal weight loss and culminated in one death. Furthermore, they also observed shRNA-dependent downregulation of three cellular miRNAs, including liver-specific miR-122, corroborating that shRNA overexpression can adversely perturb the miRNA/RNAi machinery in vivo. In line with this evidence, Ahn et al. [4] noted gradual hepatocyte death in mice treated with shRNA-expressing gutless adenoviral vectors that correlated with the buildup of mature shRNA molecules and the upregulation of miRNA-controlled hepatic genes.

Researchers who have studied organs other than the liver have described similar notions of shRNA-associated toxicities in the central nervous system (CNS) of the mouse and rat. Notable examples came from Beverly Davidson's group [5], who expressed three shRNAs plus a control against the Huntington's disease homolog in mouse striatum and observed significant neurotoxicities with two active shRNAs as well as the control shRNA. All shRNAs were expressed from the potent U6 promoter and were delivered via efficient AAV1 vectors, and toxicity correlated with shRNA abundance but not with silencing activity. In a later study, the same group again noted severe neurotoxicity with another AAV/U6-driven shRNA, this time in mouse cerebellum [6]. These conditions and findings are highly reminiscent of those in the Grimm et al. study in the liver [2], implying that nonspecific shRNA toxicity can occur in multiple cell and tissue types in vivo. Indeed, Martin et al. [7] recently inadvertently recapitulated shRNA-induced lethalities using AAV1/U6 vectors to express three distinct shRNAs in the striata of various mouse strains and validated the evidence for the involvement of shRNAinduced miRNA dysregulation.

Exemplifying that shRNA toxicity in the CNS is not species-specific, Ulusoy et al. [8] reported cytotoxicity from shRNA overexpression in the rat substantia nigra. Using AAV5 to deliver two shRNAs against tyrosine hydroxylase plus two controls, that group noted a dosedependent loss of nigral dopaminergic neurons with all four shRNAs. Similarly, Khodr and co-workers [9] reported neuron loss in the substantia nigra of rats injected with AAV2 expressing an shRNA against $\alpha$ synuclein or an irrelevant control shRNA. Moreover, Ehlert et al. [10] found a dose-dependent adverse tissue response and neuronal degeneration following AAV1mediated expression of three distinct shRNAs (including one control) in the red nucleus of rats. Lowering virus amounts, and thus shRNA expression, reduced these effects, and toxicity was absent when an inferior (as compared to AAV1) AAV5 vector was used in another cell type, together providing further support for the saturation model.

Next to rodent liver and CNS, Bish et al. [11] recently reported severe cardiac dysfunction and toxicity in three dogs treated with scAAV6-expressing anti-phospholamban shRNA. Their finding that shRNA treatment is associated with alterations in expression of two cellular miRNAs suggests that toxic oversaturation of endogenous RNAi pathways can also occur in large animals.

\section{...to first insights into the underlying cellular mechanisms...}

As noted, the recurrent correlations of cytotoxicities with shRNA abundance and miRNA dysregulation in many reports fuel the model that ectopic shRNA expression can saturate key factors in the miRNA processing pathway. Ample support for this concept is actually provided by numerous studies recapitulating the in vivo findings in cultured cells. For instance, the Chen group [12] showed that high-level, U6-driven shRNA expression from a lentiviral vector causes cytotoxicity in primary human lymphocytes, which could be relieved by encoding the same shRNA under the weaker $\mathrm{H} 1$ promoter. Likewise, Pan et al. [13] noted downregulation of hepatic miRNAs in a liver cell line infected with U6shRNA-encoding lentiviral vectors. Moreover, Khan et al. [14] conducted an extensive meta-analysis of over 150 siRNA or shRNA transfection experiments in which they described frequent upregulation of miRNA-controlled genes upon abundant siRNA and/or shRNA expression. Importantly, some of these studies also provide clues to the limiting RNAi factors. Thus far the leading suspects that have emerged from this in vitro work are Exportin-5, the nuclear karyopherin that shuttles shRNAs and miRNAs into the cytoplasm, as well as Argonaute-2, a critical RNA-induced silencing complex (RISC) component that binds and cleaves targeted mRNAs [2,15-18]. Interestingly, Bennasser and colleagues [19] recently reported that Exportin-5 saturation may also reduce Dicer expression and hence activity, adding another layer of complexity to the cellular mechanisms underlying RNAi toxicity.

Validating the potential rate-limiting nature of these factors in an in vivo setting is obviously more 
challenging, yet early reports are rapidly accumulating. In fact, hepatic Exportin-5 and Argonaute- 2 coexpression from AAV vectors was recently shown to increase shRNA potency in the livers of adult mice and to partly alleviate RNAi toxicity, implying that these two factors are also prone to exogenous saturation in vivo [2,17]. There is further indirect support in a recent study [7] for a correlation of diminished Exportin-5 levels in a particular mouse strain with an increased susceptibility to shRNA-induced neurotoxicity, and others have proposed that the relatively low Exportin-5 expression in the brain may generally render this organ particularly sensitive to adverse saturation effects [10]. Notably, despite the absence of reports to date on in vivo morbidities or deaths resulting from siRNA delivery, there is clear evidence that high intracellular siRNA abundance can also saturate critical RNAi components. For instance, the Rossi group [15] reported that transfected siRNAs can compete with each other, with cotransfected shRNAs or with endogenous miRNAs for RISC incorporation. As with shRNAs, Argonaute- 2 appears to play a crucial role in this process, as its overexpression has been shown to at least partially relieve some of these competition effects [17]. Moreover, as noted above, Khan et al. [14] found that siRNA transfection frequently perturbs cellular miRNA expression and thus regulation of endogenous gene expression. These and additional similar findings clearly imply that dose- and saturation-dependent cytotoxicity is not restricted to vector-encoded shRNAs, but can be induced and observed with siRNA delivery. That more severe effects still have not been noted in vivo may be related to the facts that (1) achieving high intracellular doses of siRNAs is difficult compared to shRNA expression and (2) the typically short-term kinetics of siRNA persistence and activity may not suffice to perturb the cellular RNAi machinery to an extent that would cause toxicity.

\section{...and to novel clinically relevant strategies to alleviate RNAi toxicity}

The available evidence to date suggests that a major goal for future clinical RNAi applications must be to thwart the risk of saturating endogenous RNAi pathways by exogenous shRNAs without compromising their therapeutic efficacy. Toward this aim, a multitude of concepts can be envisioned that fall roughly into two categories: improvements in the RNAi vector itself or advances in our understanding of cellular RNAi mechanisms (Figure 1).

The first category comprises various practical strategies that can be adopted to limit intracellular steadystate levels of ectopic RNAi substrates to tolerable yet still effective degrees. Accordingly, one approach is the use of weaker shRNA promoters, such as $\mathrm{H} 1$ or $7 \mathrm{SK}$ instead of U6, or moderate and/or tissue-specific RNA polymerase II promoters $[17,20]$. Lowering vector doses or switching to a less potent viral variant can likewise reduce shRNA expression to safer levels, especially since the therapeutic window for shRNA expression appears to be relatively wide (at least for potent molecules), frequently allowing variations in vector doses of more than two orders of magnitude without major losses in target knockdown activities $[2,8,10,17]$. However, for some applications, lowering the vector doses below a critical threshold may adversely interfere with a need for complete target tissue transduction, implying that this avenue will have to be adapted to each scenario $[6,10]$. Another useful strategy may be to express active RNAi sequences from a miRNA scaffold, which has proved particularly valuable in the CNS thus far $[5,6]$. The reasons for the presumably lower in vivo toxicity from miRNA-like vectors as compared to shRNA counterparts are still not fully clear, but the generally reduced abundance of mature miRNA strands may play a critical role. Also, since miRNAs are being shunted through an endogenous processing pathway, their entry into RISC might be slower compared to highly expressed shRNAs or transfected siRNAs. It has been proposed that this may in turn further reduce the likelihood of adverse saturation effects [15]. On the other hand, since miRNA biogenesis already begins with Drosha processing (a step that is skipped by siRNAs and shRNAs), an important question for future research will be to investigate the possibility of specific nuclear saturation events with miRNA vectors. Combining low-copy RNAi strategies with other modes of gene silencing, such as U1 inhibition or ribozymes, can also maintain high efficiency while minimizing saturation risks [21-23].

Regarding the second category, several laboratories have initiated attempts to mathematically model RNAi in mammalian cells [24,25], which will hopefully further help researchers to rationally improve the efficacy and safety of their RNAi strategies. For instance, Cuccato et al. [24] calculated the number of active RISC to be in a range from $10^{3}$ to $10^{4}$ in a typical mammalian cell and accordingly proposed that the number of ectopic RNAi triggers ideally be kept below this range to avoid saturation effects. Likewise, Arvey et al. [25] presented a mathematical model according to which the efficiency of therapeutic RNAi molecules on their intended target depends on the overall abundance of potential binding sites in the cell. Hence an important implication is that to prevent this dilution effect and to maximize siRNA or shRNA potency, sequences that have minimal off-targets should be preferred. This will not only eliminate unintended silencing effects but also permit decreasing the dose of the RNAi trigger, which will in turn minimize the risk of nonspecific saturation effects. 


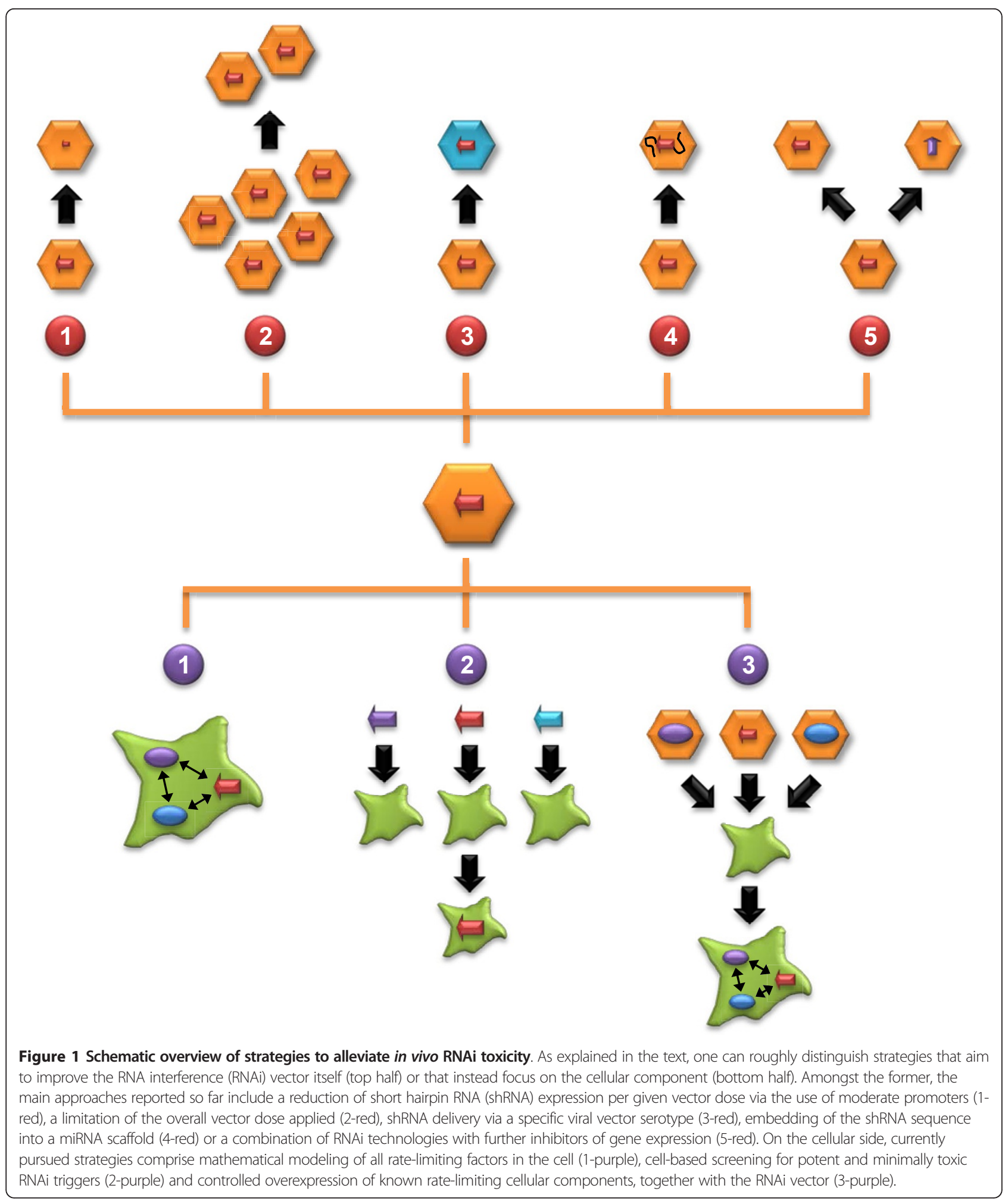

In addition, other investigators are concomitantly screening libraries of RNAi inducers in a high-throughput fashion for potent and safe molecules [26]. As with the modeling approach, their aim is to identify favorable features of RNAi triggers and unravel rate-limiting cellular steps and components to ultimately deduce guidelines for the rational design of optimized RNAi templates and strategies. Most critical here will be the 
use of appropriate model systems which take into consideration that the manifestation and extent of RNAiassociated toxicities can vary greatly with the specific cell type and depend on many other variables that may not be easy to truly mimic in vitro. An example of the latter is the observation by Beer et al. [27] that even low doses of vector-encoded shRNAs can have fatal side effects in mice that coexpress a proto-oncogene in the liver. In these animals, even marginal hepatocyte death was sufficient to accelerate tumorigenesis, a phenomenon that would have been missed in isolated cell cultures. If, however, such cultures have to be used, it may be important to employ primary human cells (where available) instead of established cell lines. This is implied by the study by An et al. [12], for instance, wherein shRNA cytotoxicity manifested only in primary lymphocytes and not in a human T-cell line. Also notable along these lines are data published by Martin et al. [7] indicating that the genetic background of rodents can modify their sensitivity to RNAi toxicity, together suggesting that selecting proper cell types and animal strains is a very important consideration in preclinical RNAi trials.

The aforementioned strategy to deliberately coexpress known rate-limiting cellular RNAi factors can boost shRNA potency and reduce toxicity [2,17], yet the longterm outcomes of this particular approach for the cell and organism remain to be studied. It is interesting to note in this context that a series of recent findings have indicated that essential parts of the RNAi machinery are inherently dysregulated in many cancers or during infections with viral pathogens [28]. This suggests that, along with the mathematical strategies described above, an important goal for future (pre-)clinical research should be quantitative delineation of the exact concentrations of all RNAi components in a given cell, of the intrinsic and extrinsic silencing triggers and of their target mRNAs so that researchers can become able to adapt and fine-tune therapeutic strategies toward maximum efficiency and minimum toxicity.

\section{Conclusion}

Regarding the pace at which the field has moved from the first notion of in vivo RNAi toxicities in 2006 to today's wealth of novel options and innovative concepts to alleviate these toxicities, and considering the rapidly increasing numbers of studies reporting the successful implementation of these avenues in animals, we can certainly stay highly optimistic that the realization of safe and potent RNAi strategies in humans remains a most realistic goal for the near future.

\section{Abbreviations}

AAV: adeno-associated virus; CNS: central nervous system; miRNA: microRNA; RNAi: RNA interference; scAAV: self-complementary adeno-associated virus; shRNA: short hairpin RNA; siRNA: small interfering RNA.

\section{Acknowledgements}

The author appreciates support for this work from the Cluster of Excellence CellNetworks at the University of Heidelberg and the Chica and Heinz Schaller Foundation. Moreover, I am indebted to Kathleen Börner and Stefan Mockenhaupt for critical reading of the manuscript. I apologize to all authors who made important contributions to the subject of this article but whose work could not be referenced because of space constraints.

\section{Competing interests}

The author declares that he has no competing interests.

Received: 21 September 2011 Accepted: 26 October 2011

Published: 26 October 2011

\section{References}

1. Fire A, Xu S, Montgomery MK, Kostas SA, Driver SE, Mello CC: Potent and specific genetic interference by double-stranded RNA in Caenorhabditis elegans. Nature 1998, 391:806-811.

2. Grimm D, Streetz KL, Jopling $C L$, Storm $T A$, Pandey $K$, Davis $C R$, Marion $P$, Salazar F, Kay MA: Fatality in mice due to oversaturation of cellular microRNA/short hairpin RNA pathways. Nature 2006, 441:537-541.

3. Borel F, van Logtenstein R, Koornneef A, Maczuga P, Ritsema T, Petry H, van Deventer SJH, Jansen PL, Konstantinova P: In vivo knock-down of multidrug resistance transporters $A B C C 1$ and $A B C C 2$ by $A A V$-delivered shRNAs and by artificial miRNAs. J RNAi Gene Silencing 2011, 7:434-442.

4. Ahn M, Witting SR, Ruiz R, Saxena R, Morral N: Constitutive expression of shRNA in vivo triggers build up of mature hairpin molecules. Hum Gene Ther 2011.

5. McBride JL, Boudreau RL, Harper SQ, Staber PD, Monteys AM, Martins I, Gilmore BL, Burstein H, Peluso RW, Polisky B, Carter BJ, Davidson BL: Artificial miRNAs mitigate shRNA-mediated toxicity in the brain: implications for the therapeutic development of RNAi. Proc Natl Acad Sci USA 2008, 105:5868-5873.

6. Boudreau RL, Martins I, Davidson BL: Artificial microRNAs as siRNA shuttles: improved safety as compared to shRNAs in vitro and in vivo. Mol Ther 2009, 17:169-175.

7. Martin JN, Wolken N, Brown T, Dauer WT, Ehrlich ME, Gonzalez-Alegre P: Lethal toxicity caused by expression of shRNA in the mouse striatum: implications for therapeutic design. Gene Ther 2011, 18:666-673.

8. Ulusoy A, Sahin G, Björklund T, Aebischer P, Kirik D: Dose optimization for long-term rAAV-mediated RNA interference in the nigrostriatal projection neurons. Mol Ther 2009, 17:1574-1584.

9. Khodr CE, Sapru MK, Pedapati J, Han Y, West NC, Kells AP, Bankiewicz KS, Bohn MC: An a-synuclein AAV gene silencing vector ameliorates a behavioral deficit in a rat model of Parkinson's disease, but displays toxicity in dopamine neurons. Brain Res 2011, 1395:94-107.

10. Ehlert EM, Eggers R, Niclou SP, Verhaagen J: Cellular toxicity following application of adeno-associated viral vector-mediated RNA interference in the nervous system. BMC Neurosci 2010, 11:20.

11. Bish LT, Sleeper MM, Reynolds C, Gazzara J, Withnall E, Singletary GE, Buchlis G, Hui D, High KA, Gao G, Wilson JM, Sweeney HL: Cardiac gene transfer of short hairpin RNA directed against phospholamban effectively knocks down gene expression but causes cellular toxicity in canines. Hum Gene Ther 2011, 22:969-977.

12. An DS, Qin FX, Auyeung VC, Mao SH, Kung SK, Baltimore D, Chen IS: Optimization and functional effects of stable short hairpin RNA expression in primary human lymphocytes via lentiviral vectors. Mol Ther 2006, 14:494-504.

13. Pan $Q$, de Ruiter PE, von Eije KJ, Smits R, Kwekkeboom J, Tilanus HW, Berkhout $\mathrm{B}$, Janssen $\mathrm{HL}$, van der Laan $\mathrm{L}$ : Disturbance of the microRNA pathway by commonly used lentiviral shRNA libraries limits the application for screening host factors involved in hepatitis $C$ virus infection. FEBS Lett 2011, 585:1025-1030.

14. Khan AA, Betel D, Miller ML, Sander C, Leslie CS, Marks DS: Transfection of small RNAs globally perturbs gene regulation by endogenous microRNAs. Nat Biotechnol 2009, 27:549-555.

15. Castanotto D, Sakurai K, Lingeman R, Li H, Shively L, Aagaard L, Soifer H, Gatignol A, Riggs A, Rossi Jj: Combinatorial delivery of small interfering RNAs reduces RNAi efficacy by selective incorporation into RISC. Nucleic Acids Res 2007, 35:5154-5164. 
16. Diederichs S, Jung S, Rothenberg SM, Smolen GA, Mlody BG, Haber DA: Coexpression of Argonaute-2 enhances RNA interference toward perfect match binding sites. Proc Natl Acad Sci USA 2008, 105:9284-9289.

17. Grimm D, Wang L, Lee JS, Schurmann N, Gu S, Borner K, Storm TA, Kay MA: Argonaute proteins are key determinants of RNAi efficacy, toxicity, and persistence in the adult mouse liver. J Clin Invest 2010, 120:3106-3119.

18. Yi R, Doehle BP, Qin Y, Macara IG, Cullen BR: Overexpression of Exportin 5 enhances RNA interference mediated by short hairpin RNAs and microRNAs. RNA 2005, 11:220-226.

19. Bennasser Y, Chable-Bessia C, Triboulet R, Gibbings D, Gwizdek C, Dargemont C, Kremer EJ, Voinnet O, Benkirane M: Competition for XPO5 binding between Dicer mRNA, pre-miRNA and viral RNA regulates human Dicer levels. Nat Struct Mol Biol 2011, 18:323-327.

20. Giering JC, Grimm D, Storm TA, Kay MA: Expression of shRNA from a tissue-specific pol II promoter is an effective and safe RNAi therapeutic. Mol Ther 2008, 16:1630-1636.

21. Grimm D, Kay MA: Combinatorial RNAi: a winning strategy for the race against evolving targets? Mol Ther 2007, 15:878-888.

22. Abad X, Razquin N, Abad A, Fortes P: Combination of RNA interference and $\mathrm{U} 1$ inhibition leads to increased inhibition of gene expression. Nucleic Acids Res 2010, 38:e136.

23. DiGiusto DL, Krishnan A, Li L, Li H, Li S, Rao A, Mi S, Yam P, Stinson S, Kalos M, Alvarnas J, Lacey SF, Yee JK, Li M, Couture L, Hsu D, Forman SJ, Rossi JJ, Zaia JA: RNA-based gene therapy for HIV with lentiviral vectormodified $\mathrm{CD} 34^{+}$cells in patients undergoing transplantation for AIDSrelated lymphoma. Sci Trans/ Med 2010, 2:36ra43.

24. Cuccato G, Polynikis A, Siciliano V, Graziano M, di Bernardo M, di Bernardo D: Modeling RNA interference in mammalian cells. BMC Syst Biol 2011, 5:19.

25. Arvey A, Larsson E, Sander C, Leslie CS, Marks DS: Target mRNA abundance dilutes microRNA and siRNA activity. Mol Syst Biol 2010, 6:363.

26. Li Z, Fortin Y, Shen SH: Forward and robust selection of the most potent and noncellular toxic siRNAs from RNAi libraries. Nucleic Acids Res 2009, 37:e8.

27. Beer S, Bellovin DI, Lee JS, Komatsubara K, Wang LS, Koh H, Börner K, Storm TA, Davis CR, Kay MA, Felsher DW, Grimm D: Low-level shRNA cytotoxicity can contribute to MYC-induced hepatocellular carcinoma in adult mice. Mol Ther 2010, 18:161-170.

28. Mockenhaupt S, Schurmann N, Grimm D: When cellular networks run out of control: global dysregulation of the RNAi machinery in human pathology and therapy. Prog Mol Biol Transl Sci 2011, 102:165-242.

doi:10.1186/1758-907X-2-8

Cite this article as: Grimm: The dose can make the poison: lessons learned from adverse in vivo toxicities caused by RNAi overexpression. Silence 2011 2:8.

\section{Submit your next manuscript to BioMed Central and take full advantage of:}

- Convenient online submission

- Thorough peer review

- No space constraints or color figure charges

- Immediate publication on acceptance

- Inclusion in PubMed, CAS, Scopus and Google Scholar

- Research which is freely available for redistribution

Submit your manuscript at www.biomedcentral.com/submit
C Biomed Central 